\title{
Visual search in spatial neglect studied with a preview paradigm
}

\section{Julia Fellrath ${ }^{1,2 *}$, Vanessa Blanche-Durbec ${ }^{3}$, Armin Schnider $^{1,2}$, Anne-Sophie Jacquemoud ${ }^{1}$ and Radek Ptak ${ }^{1,2 *}$}

1 Division of Neurorehabilitation, University Hospitals Geneva, Geneva, Switzerland

${ }^{2}$ Faculty of Medicine, Laboratory of Cognitive Neurorehabilitation, University of Geneva, Geneva, Switzerland

${ }^{3}$ Service Universitaire de Psychiatrie de l'Âge Avancé, CHUV, Route du Mont, Lausanne, Switzerland

\section{Edited by:}

Mario Bonato, University of Padova,

Italy

\section{Reviewed by:}

Ray Klein, Dalhousie University, Canada

Ana B. Chica, University of Granada, Spain

\section{*Correspondence:}

Julia Fellrath and Radek Ptak,

Division of Neurorehabilitation,

University Hospitals Geneva, 26, av. de Beau-Séjour, 1211 Geneva 14, Switzerland.

e-mail: julia.fellrath@hcuge.ch; radek.ptak@hcuge.ch
Impaired visual search is a hallmark of spatial neglect. When searching for an unique feature (e.g., color) neglect patients often show only slight visual field asymmetries. In contrast, when the target is defined by a combination of features (e.g., color and form) they exhibit a severe deficit of contralesional search. This finding suggests a selective impairment of the serial deployment of spatial attention. Here, we examined this deficit with a preview paradigm. Neglect patients searched for a target defined by the conjunction of shape and color, presented together with varying numbers of distracters. The presentation time was varied such that on some trials participants previewed the target together with same-shape/different-color distracters, for 300 or $600 \mathrm{~ms}$ prior to the appearance of additional different-shape/same-color distracters. On the remaining trials the target and all distracters were shown simultaneously. Healthy participants exhibited a serial search strategy only when all items were presented simultaneously, whereas in both preview conditions a pop-out effect was observed. Neglect patients showed a similar pattern when the target was presented in the right hemifield. In contrast, when searching for a target in the left hemifield they showed serial search in the no-preview condition, as well as with a preview of $300 \mathrm{~ms}$, and partly even at $600 \mathrm{~ms}$. A control experiment suggested that the failure to fully benefit from item preview was probably independent of accurate perception of time. Our results, when viewed in the context of existing literature, lead us to conclude that the visual search deficit in neglect reflects two additive factors: a biased representation of attentional priority in favor of ipsilesional information and exaggerated capture of attention by ipsilesional abrupt onsets.

Keywords: visual search, pop-out, saliency, selective attention, spatial neglect, temporal processing, parietal lobe

\section{INTRODUCTION}

Impaired visual search is one of the primary characteristics of spatial neglect. Patients with this disorder may fail to find personal belongings or may bump into obstacles when these are presented in contralesional space (Halligan and Marshall, 1993; Milner and McIntosh, 2005). Consequently, visual search tasks are among the most sensitive tests of spatial neglect. Several reports have shown that the visual search deficit of neglect patients varies as a function of the extent to which the task engages serial or parallel mechanisms of spatial attention. These studies were strongly influenced by work with healthy participants showing that performance in visual search for targets defined by a unique feature (feature search, e.g., a red $O$ among red Xs) is largely independent of the number of distracters (Treisman and Gelade, 1980; Treisman and Gormican, 1988) whereas when the target is defined by a unique combination of features (conjunction search, e.g., a red $O$ among red $X$ s and green $O s$ ) search times linearly increase with increasing numbers of distracters (Treisman and Gormican, 1988). Feature search is effortless and the target automatically "pops-out" among the distracters, suggesting that the search items are examined pre-attentively and in parallel. Conjunction search is effortful and search times depend on the number of distracters, suggesting that they are processed attentively and serially (Bricolo et al., 2002). The time necessary to examine an individual item in the display (i.e., the search rate) is expressed as the slope of a simple regression computed with search times for targets embedded in displays with increasing display size. In pop-out search the search rate approaches zero whereas in serial search it is in the order of several tens of milliseconds (Treisman and Gormican, 1988).

Functional imaging and virtual lesion studies using transcranial magnetic stimulation indicate that the posterior parietal cortex (PPC) plays a special role in feature binding and conjunction search (Corbetta et al., 1995; Ashbridge et al., 1997). This is confirmed by several reports of patients with damage to PPC exhibiting visual binding deficits in the form of illusory conjunctions (Cohen and Rafal, 1991; Friedman-Hill et al., 1995). Previous visual search studies examined the question whether the contralesional impairment of spatial attention characterizing neglect affects serial and parallel search mechanisms to the 
same degree, or whether pre-attentive processing - as is required in search for pop-out targets - is preserved. However, these studies have produced equivocal results. Regarding serial search for feature combinations, most studies agree that neglect patients have much slower search rates for contralesional targets (Riddoch and Humphreys, 1987; Humphreys and Riddoch, 1993; Aglioti et al., 1997; Esterman et al., 2000). In contrast, while some studies found that search for pop-out targets is impaired in the contralesional visual field (Eglin et al., 1989; Pavlovskaya et al., 2002; Behrmann et al., 2004; Eramudugolla and Mattingley, 2009), others reported intact performance (Aglioti et al., 1997; Esterman et al., 2000).

Several factors might account for these differences. Ipsilesional distracter stimuli strongly capture attention of patients with neglect (Posner et al., 1984; Morrow and Ratcliff, 1988; Golay et al., 2005), and this effect is particularly strong when distracters share perceptual properties with the search target (Ptak and Schnider, 2006). Some studies have shown that the number of ipsilesional distracters strongly affects visual search for a contralesional target (Eglin et al., 1989; Grabowecky et al., 1993), suggesting that impaired attentional disengagement might underlie deficient visual search performance (Posner et al., 1984; Bonato et al., 2009; Schnider et al., 2011). Peru and Chelazzi (2008) proposed that visual search in neglect is better described as result of interactions between a focused or distributed mode of processing rather than by the distinction between pre-attentive and attentive mechanisms. According to this proposal, patients with slight forms of neglect have difficulty directing focused attention to the contralesional hemifield while patients with severe neglect have an additional ipsilesional bias preventing distribution of attention across the hemifields. Finally, differences in search performance of patients with neglect might also be related to the anatomical location of brain damage. Thus, neglect patients with damage involving the inferior temporal cortex show particularly slow search for contralesional conjunction targets (Ptak and Valenza, 2005).

Reconciling these different proposals is difficult, as feature and conjunction search likely rely on distinct, but partially overlapping attentional mechanisms. Rather than using distinct feature and conjunction tasks we investigated the underlying attentional processes by presenting the different items of a conjunction display separated in time. We showed neglect patients a feature display and added supplementary distracters following a variable preview period, which transformed the display into a conjunction display. This is a variant of the preview paradigm (Olivers and Humphreys, 2004) in which participants are shown a preview of a set of distracters (e.g., green $H$ s) some time (e.g., $1000 \mathrm{~ms}$ ) prior to adding the second set of items (e.g., blue $A s$ ), which includes the target (blue $H$ ). Thus, apart from the preview period the task conforms to a standard conjunction search task. Nevertheless, healthy participants exhibit search rates compatible with fast, parallel search, indicating that the preview period effectively reduces the task to a feature search task.

In the present study the target (a green $T$ ) was presented together with the previewed items (red Ts), before adding the remaining distracters (green $L s$ ). Thus, during the preview period the task was a standard feature task, and it only became a conjunction task once the remaining distracters were added. The search rate (expressed as a function of preview duration) in the modified preview task is therefore an indicator of the time needed to activate pre-attentive search mechanisms.

\section{MATERIALS AND METHODS PARTICIPANTS}

Nine patients (three females) with left spatial neglect following recent right-hemispheric brain injury and 13 neurologically healthy control participants (eight females) participated in this study. Approval was obtained from the ethical committee of the University Hospital Geneva, and all participants gave written informed consent. The demographic data and results of clinical testing are presented in Table 1. Control participants and neglect patients had comparable age $\left(t_{20}=0.89\right)$, and all but one ambidextrous control subject were right-handed. All neglect patients manifested behavioral symptoms of visual neglect (e.g., unawareness of persons or objects placed contralesionally; difficulty with dressing, eating, grooming etc.) as well as objective neglect signs in at least three out of five neglect tests: the "Bells" cancellation test (Gauthier et al., 1989), cancellation of inverted among upright Ts (Ptak et al., 2007), line bisection (Schenkenberg et al., 1980) and sentence copying (Wilson et al., 1987). Patients had preserved visual fields, as assessed on clinical confrontation and/or computerized perimetry (white dot presented on black background).

\section{VISUAL SEARCH TASK}

In the visual search task participants were required to search for a green $T$ presented on black background among varying numbers of distracters (green $L$ s and red $T$ s). The search displays were constructed by plotting letters on an imaginary circle (diameter: $11.4^{\circ}$ ), divided in sixteen equal sectors (Figure 1). Letters, uppercase $L$ and $T$, were bright red (RGB-values: $255,0,0)$ or green (RGB-values: $0,255,0$ ), and were $0.76^{\circ}$ high $\times 0.67^{\circ}$ wide. Search displays contained 4, 8, or 16 elements. On target-present trials (two thirds of all trials) one of these was the target (green $T$ ) and the remainder distracters; on target-absent trials all search elements were distracters. On $67 \%$ of all target-present trials the target was presented at position 4 in the right visual field (RVF) or position 12 in the left visual field (LVF). On the remaining trials it could appear at one of the positions $2,6,8,10,14$ or 16 , selected randomly, while distracters could appear at all positions. Thus, when the search display contained 16 elements, all positions were occupied, while when it contained 4 or 8 elements positions were selected randomly with the constraint that at least two items appeared in each hemifield.

Stimuli were presented on a $15^{\prime}$ laptop screen running at a resolution of $1280 \times 768$ pixels, placed at a distance of $60 \mathrm{~cm}$ from the participant. In the preview conditions, the search display was separated into a first display (preview) and a second display (final view). The preview display contained the target as well as all distracters that differed from the target only by their color (i.e., red $T s)$. The final view contained distracters that differed from the target by their form (i.e., green Ls). In the no-preview condition the target and all distracters were presented simultaneously. Each trial started with the presentation of a white fixation cross 
Table 1 | Demographic and clinical characteristics of neglect patients and control participants.

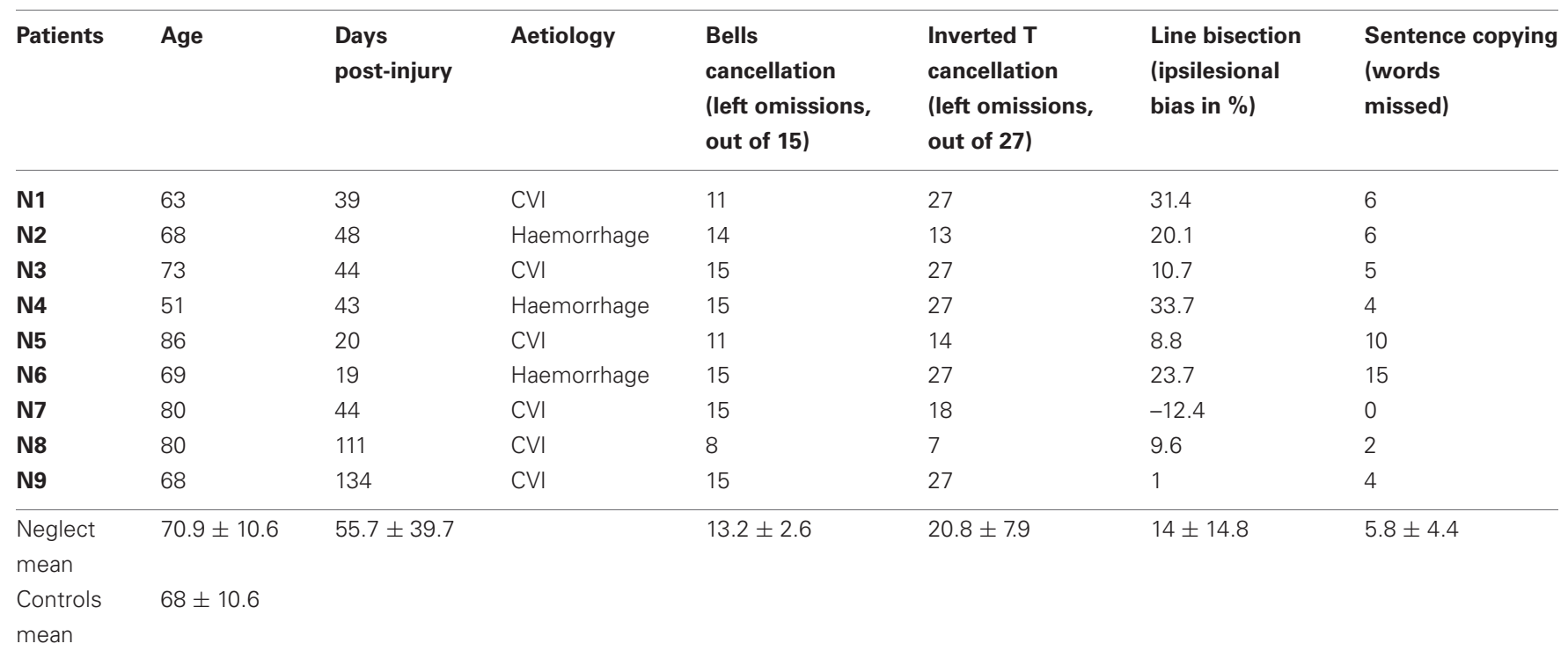

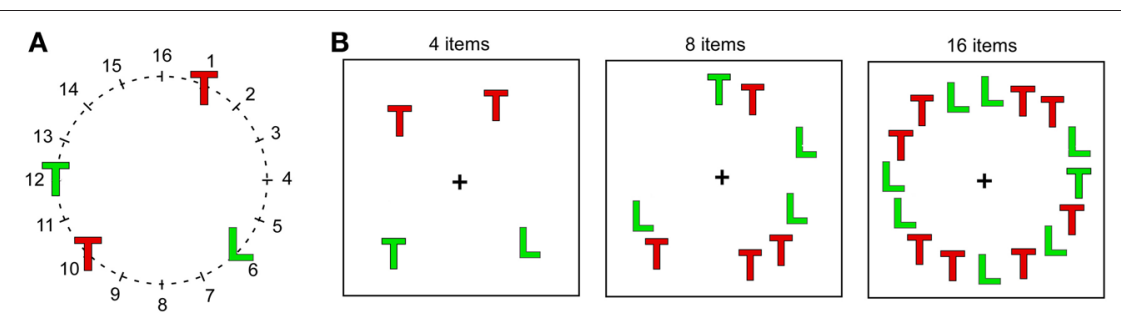

FIGURE 1 | Displays used in the visual search task. (A) Schematic presentation of the 16 positions on an imaginary circle, on which search items were presented. (B) Three examples of search displays with different display sizes and the target (green T) presented at different positions. Note that in reality letters were shown on black background and that their size is exaggerated in comparison to the circle. in the middle of the screen. After 1000 ms either the final display appeared (no-preview condition) or a preview display, followed after $300 \mathrm{~ms}$ or $600 \mathrm{~ms}$ by the final display. In both preview conditions the task was therefore reduced to a color search task and only became a conjunction search task once the preview period ended. The final display stayed on until there was a response, or for a maximum duration of $5000 \mathrm{~ms}$. Thus, the design of the task was a 2 (Target Position: $L V F, R V F$ ) $\times 3$ (Display Size: 4, 8, 16 items) $\times 3$ (Preview Condition: 0, 300, $600 \mathrm{~ms}$ ) factor experiment. Stimuli were presented in blocks of 63 trials consisting of two trials per Target Position $\times$ Display Size $\times$ Preview Condition cell, nine trials with the target-presented randomly at other than the left/right positions, and 18 target-absent trials.

Participants were instructed to press the space bar as soon as they detected a green $T$ and to withhold reaction when the target was absent. Control participants completed 10 blocks, resulting in 20 trials per cell; neglect patients completed up to 30 blocks, resulting in up to 60 trials per cell.

\section{TEMPORAL JUDGMENT TASK}

Three neglect patients (N7, N8, N9) and seven controls (C7-C13) were tested in a temporal judgment task, which examined participants' perception of the temporal order of events. The experimental setup was the same as in the visual search task, with two important modifications. First, only target-present displays were shown. Second, participants were informed that some items of the display might appear earlier than the remaining items. Their task was to indicate whether all items were presented at the same time, or whether the target $T$ was presented prior to a subset of distracters. Participants gave their answer orally, and the experimenter registered the answer by pressing on one of two different keyboard buttons. Each participant completed at least ten blocks of 45 trials, resulting in at least 20 trials per Target Position $\times$ Display Size $\times$ Preview Condition cell.

\section{RESULTS \\ RESPONSE ACCURACY}

The number of false alarms was very low in both groups (1.04 and $2.78 \%$ in controls and neglect patients, respectively) and was therefore not analyzed. Table 2 shows the percent targets missed by control participants and neglect patients. Omission rates were close to zero in the control group and were therefore not analyzed. Across conditions neglect patients missed on average $0.8-7.4 \%$ ipsilesional targets. Not surprisingly their omission rates were much higher for contralesional targets (between $11.6-34 \%)$. These results were analyzed with repeated-measures 
Table 2 | Average percent missed targets in the control and neglect group as a function of target position (LVF, RVF), display size (4, 8 or 16 items) and preview condition $(0,300$ or $600 \mathrm{~ms})$.

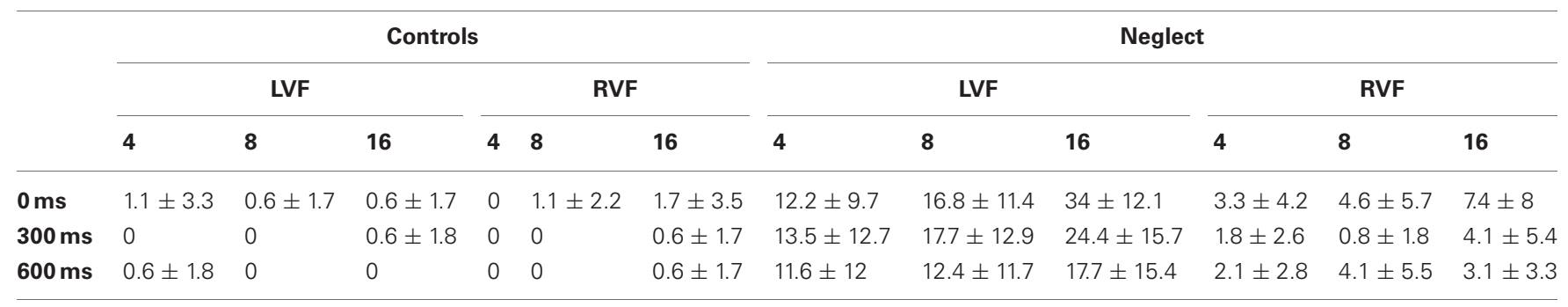

ANOVA with the factors Target Position, Display Size and Preview Condition. Significant effects were followed-up computing post-hoc pairwise comparisons (Fisher test) with Bonferroniadjusted level of significance. The analysis revealed significant main effects of Target Position $\left[F_{(1,8)}=30.25, P<0.001\right]$, Display Size $\left[F_{(2,16)}=16.62, P<0.001\right]$ and Preview Condition $\left[F_{(2,16)}=13.55, P<0.001\right]$, as well as significant interactions of Target Position $\times$ Display Size $\left[F_{(2,16)}=10.83, P<0.01\right]$, Target Position $\times$ Preview Condition $\left[F_{(2,16)}=3.99, P<0.05\right]$ and Display Size $\times$ Preview Condition $\left[F_{(4,32)}=8.05, P<0.001\right]$. We did not analyze these effects further because of the presence of a significant three-way interaction of Target Position $\times$ Display Size $\times$ Preview Condition $\left[F_{(4,32)}=3.11, P<0.05\right]$. Post-hoc comparisons revealed that whereas there was no difference across conditions for target omissions in the RVF, the percentage of LVF omissions significantly increased with increasing display size, but only in preview conditions 0 and 300. Thus, neglect patients found the search task hardest when the target was presented in the LVF, when many distracters were present, and when all display items were presented simultaneously or with a short preview. As will be seen in the following section this pattern is similar to the pattern of reaction times (RTs) and the findings can therefore not be explained by a speed-accuracy trade-off.

\section{REACTION TIME}

Before analyzing possible effects of search conditions on RT, we examined the possible contribution of a bias resulting from the fact that targets appeared more often at positions 4 (right) and 12 (left) than any other position (this constraint being introduced in order to limit the number of experimental trials). We argued that, if participants were influenced by the biased probability of target occurrence, their omission rates and RTs for the most frequent positions of the target would gradually decrease. In order to test this prediction, we analyzed RTs of neglect patients to all LVF and RVF targets across 10 experimental blocks. Repeated-measures ANOVAs did not reveal any change in omission rates (LVF: $\left[F_{(9,72)}=0.58\right]$; RVF: $\left.\left[F_{(9,72)}=1.22\right)\right]$ or RTs $\left(\operatorname{LVF}:\left[F_{(9,72)}=\right.\right.$ 1.34]; RVF: $\left.\left[F_{(9,72)}=1.94\right)\right]$ in neglect patients. Though this finding does not definitely exclude a bias due to different location probabilities, it suggests that the contribution of such a bias was negligible.

As Figure 2 shows the pattern of RTs to ipsilesional targets is comparable between groups. In contrast, neglect patients were differently affected by search conditions when searching for contralesional targets.
In an initial analysis, results were submitted to a mixed ANOVA with Group (control, neglect), Target Position, Display Size and Preview Condition as factors. This analysis revealed significant main effects of Group $\left[F_{(1,16)}=23.23, P<0.001\right]$, Target Position $\left[F_{(2,16)}=23.36, P<0.001\right]$, Display Size $\left[F_{(2,32)}=51.11, P<0.001\right]$, and Preview Condition $\left[F_{(2,32)}=\right.$ 107.31, $P<0.0001]$. All two-way and three-way interactions were significant: Group $\times$ Target Position $\left[F_{(1,16)}=28.67\right.$, $P<0.0001]$, Group $\times$ Display Size $\left[F_{(2,32)}=7.98, P<0.01\right]$, Group $\times$ Preview Condition $\left[F_{(2,32)}=7.87, P<0.01\right]$, Target Position $\times$ Display Size $\left[F_{(2,32)}=9.65, P<0.001\right]$, Target Position $\times$ Preview Condition $\left[F_{(2,32)}=5.99, \quad P<0.01\right]$, Display Size $\times$ Preview Condition $\left[F_{(4,64)}=58.71, P<0.0001\right]$, Group $\times$ Target Position $\times$ Display Size $\left[F_{(2,32)}=16.71\right.$, $P<0.0001]$, Group $\times$ Target Position $\times$ Preview Condition $\left[F_{(2,32)}=16.98, P<0.0001\right]$, Group $\times$ Display Size $\times$ Preview Condition $\left[F_{(4,64)}=6.60, P<0.001\right]$, and Target Position $\times$ Display Size $\times$ Preview Condition $\left[F_{(4,64)}=8.18, P<0.0001\right]$. Finally, the four-way interaction between all factors was also significant $\left[F_{(4,64)}=17.96, P<0.0001\right]$.

In order to better understand this complex pattern, we decided to follow up these results with separate repeated-measures analyzes of RTs to LVF and RVF targets, focusing on the factors Group, Display Size and Preview Condition.

For targets presented in the RVF this analysis revealed significant effects of Display Size $\left[F_{(2,32)}=49.4, P<0.0001\right]$ and Preview Condition $\left[F_{(2,32)}=66.48, P<0.0001\right]$, as well as an interaction between these two factors $\left[F_{(4,64)}=29.59, P<\right.$ 0.0001]. Pairwise comparisons showed that in both preview conditions RTs were comparable across different display sizes, but increased from display size 4-16 when all items were presented simultaneously. In addition, RTs were longer in the no-preview condition compared to both preview conditions for all three display sizes. The main result of these comparisons was that no interaction with the factor Group reached significance, indicating that in the RVF neglect patients had a pattern of results comparable to healthy participants.

The same analysis on RTs to LVF items revealed significant effects of Group $\left[F_{(1,16)}=32.46, P<0.0001\right]$, Display Size $\left[F_{(2,32)}=30.89, P<0.0001\right]$ and Preview Condition $\left[F_{(2,32)}=\right.$ 67.95, $P<0.0001]$. The two-way interactions of Group $\times$ Display Size $\left[F_{(2,32)}=12.95, P<0.0001\right]$, Group $\times$ Preview Condition $\left[F_{(2,32)}=16.92, P<0.0001\right]$ and Display Size $\times$ Preview Condition $\left[F_{(4,64)}=37.4, P<0.0001\right]$ were significant. Most importantly, the three-way interaction of Group $\times$ Display 

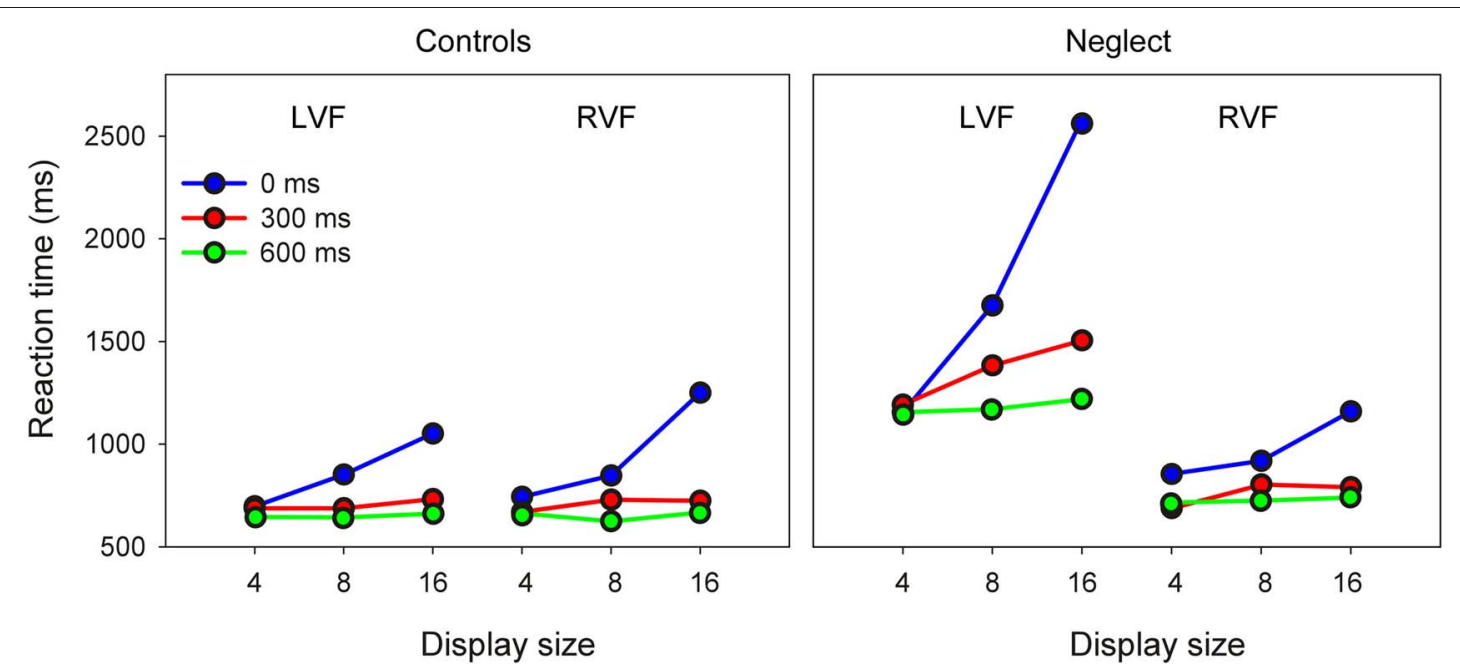

FIGURE 2 | Mean reaction time of controls and neglect patients as a function of target position, display size and preview condition (LVF/RVF: left/right visual field).

Size $\times$ Preview Condition was significant $\left[F_{(4,64)}=15.12, P<\right.$ 0.0001]. Pairwise comparisons showed for the control group very similar results as for RVF targets: RTs increased with increasing display size only when all items were presented simultaneously, and RTs were longer in this condition compared to both preview conditions at display sizes 8 and 16. The same differences were also found for the data of neglect patients. However, in addition patients showed also a significant increase of RTs in the $300 \mathrm{~ms}$ preview condition when the display contained 16 items compared to when it contained only four items. Only when the preview was as long as $600 \mathrm{~ms}$ were RTs independent of display size.

Thus, the main finding of these analyzes is that visual search of healthy participants depended on display size only when all items were presented simultaneously, while previewing the target for $300 \mathrm{~ms}$ or more was sufficient to turn the task into a pop-out task. This pattern was comparable for items shown in the LVF and RVF. In contrast, neglect patients showed a clear difference between visual fields: their search RT was independent of display size in both preview conditions when the target was shown in the RVF, while for LVF targets it was only independent of display size when the preview was $600 \mathrm{~ms}$. These effects of item preview were further examined with analyzes of search rate.

\section{SEARCH RATE}

Figure 3 shows the mean search rate of controls and neglect patients as a function of preview condition. The search rate is the time necessary to examine one individual item in conditions when the target is present (having used a go-nogo task we did not sample search times for target-absent trials and were therefore unable to compute search rates for these trials). Search rates close to zero indicate that search time is independent of the number of items in the display - a marker of pop-out search.

A mixed ANOVA revealed significant main effects of Group $\left[F_{(1,16)}=16.24, P<0.001\right]$, Target Position $\left[F_{(1,16)}=16.83\right.$, $P<0.001]$ and Preview Condition $\left[F_{(2,32)}=110.51, P<\right.$

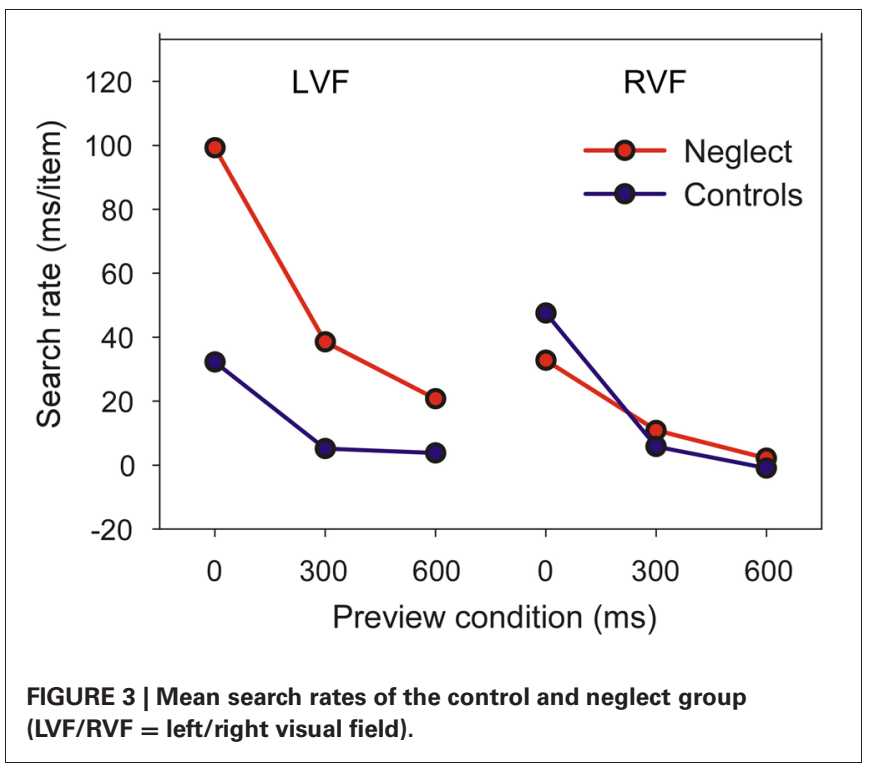

0.0001]. The two-way interaction between Group and Target Position was also significant $\left[F_{(1,16)}=25.11, P<0.001\right]$, but was not further analyzed because of the presence of a significant three-way interaction $\left[F_{(2,32)}=16.2, P<0.0001\right]$. Pairwise comparisons showed that the search rate of neglect patients was comparable to healthy controls when the target was presented in the RVF, irrespective of preview condition. In contrast, for targets in the LVF neglect patients had slower search rates than controls in the no-preview condition or when the preview was $300 \mathrm{~ms}$. However, even at the longest preview interval neglect patients seemed to benefit less from the preview than controls. We therefore performed additional paired $t$-tests evaluating whether search rates reliably differed from zero. For control participants, this was the case only when all items were presented simultaneously (LVF: $t_{8}=6.1, P<0.0001$; RVF: $t_{8}=10.6, P<0.0001$ ). 
Similarly, neglect patients had a search rate significantly greater than zero when all items were presented simultaneously (LVF, mean 99 ms: $t_{8}=10.7, P<0.0001$; RVF, mean 33 ms: $t_{8}=7.15$, $P<0.0001)$. However, in contrast to healthy participants their slope also differed from zero when the target was presented in the LVF and was previewed for $300 \mathrm{~ms}$ (mean $39 \mathrm{~ms}: t_{8}=3.39$, $P<0.01)$.

Together, these findings show that while visual search of neglect patients for RVF targets is comparable to healthy participants, patients benefit less from item preview when targets are shown in the LVF.

\section{TEMPORAL JUDGMENT}

In order to examine to what extent the preview benefit might depend on the explicit recognition of temporal separation, three neglect patients (N7, N8, N9) and seven age-matched controls (C7-C13) were tested in a temporal judgment task. The results of these subgroups in the visual search task were similar to the whole group. Figure 4 shows the mean percentage of trials on which the target was judged being presented simultaneously with all distracters. As the figure shows, the performance of control participants in this "slow" task was close to ceiling, and made it therefore difficult to compare with performance of neglect patients. We therefore asked six additional healthy participants (four females; mean age, 27 years) to make judgments of simultaneity using much shorter time intervals ("fast" task: 15 and 30, instead of 300 and $600 \mathrm{~ms}$ ). Performance of neglect patients was profoundly impaired, and differed in several respects compared to healthy participants. First, patients made more "simultaneous" judgments when the number of display items increased (Friedman test, no-preview condition, LVF: $\chi^{2}=6.0, P<0.05$; RVF: $\left.\chi^{2}=4.67, P=0.097\right)$. This appeared to be due to a generalized response bias that affected temporal judgments independently of preview condition, and was not beneficial for performance. This conclusion is supported by the observation that healthy participants performing the much more difficult "fast" task were positively influenced by item number: their performance was better with increasing display size in the no-preview condition as well as the $30 \mathrm{~ms}$ preview condition.

Second, neglect patients' temporal judgments were much less influenced by preview condition than controls. In the "slow" task healthy controls had a ratio of "simultaneous" judgments close to $0 \%$ in both preview conditions and $100 \%$ in the nopreview condition. A similar pattern was found in the "fast" task, when the $30 \mathrm{~ms}$ preview condition was compared with the no-preview condition. Only in the $15 \mathrm{~ms}$ preview condition were healthy participants' judgments of simultaneity close to chance performance. In comparison, the difference between preview and no-preview conditions was much less for neglect patients. We analyzed these data by computing average scores for preview and no-preview conditions across all display sizes, and compared these within each group with non-parametric tests. Control participants made more "simultaneous" judgments for no-preview items than preview items in the "slow" task (Wilcoxon Test, LVF: $Z=2.37, P<0.05$; RVF: $Z=2.36, P<0.05)$ and the "fast" task (LVF: $Z=2.20, P<0.05$; RVF: $Z=2.2, P<0.05$ ) whereas there was no effect of preview on neglect patients' judgments of simultaneity (LVF: $Z=1.6$; RVF: $Z=1.6$ ). Finally, in strong contrast to the visual search task neglect patients showed comparable performance for targets presented in the LVF and targets presented in the RVF (Wilcoxon test, average across all conditions: $Z=0)$.

\section{DISCUSSION}

The use of a preview paradigm to study visual search reveals several characteristics of search performance in patients with spatial neglect. When all items were presented simultaneously and the task corresponded to a conjunction search task, control participants exhibited search rates reflecting serial search. In contrast, when there was a preview of parts of the search display, their average search rate approached zero milliseconds in both preview conditions, suggesting parallel search. Neglect patients showed comparable performance when searching for targets in the right

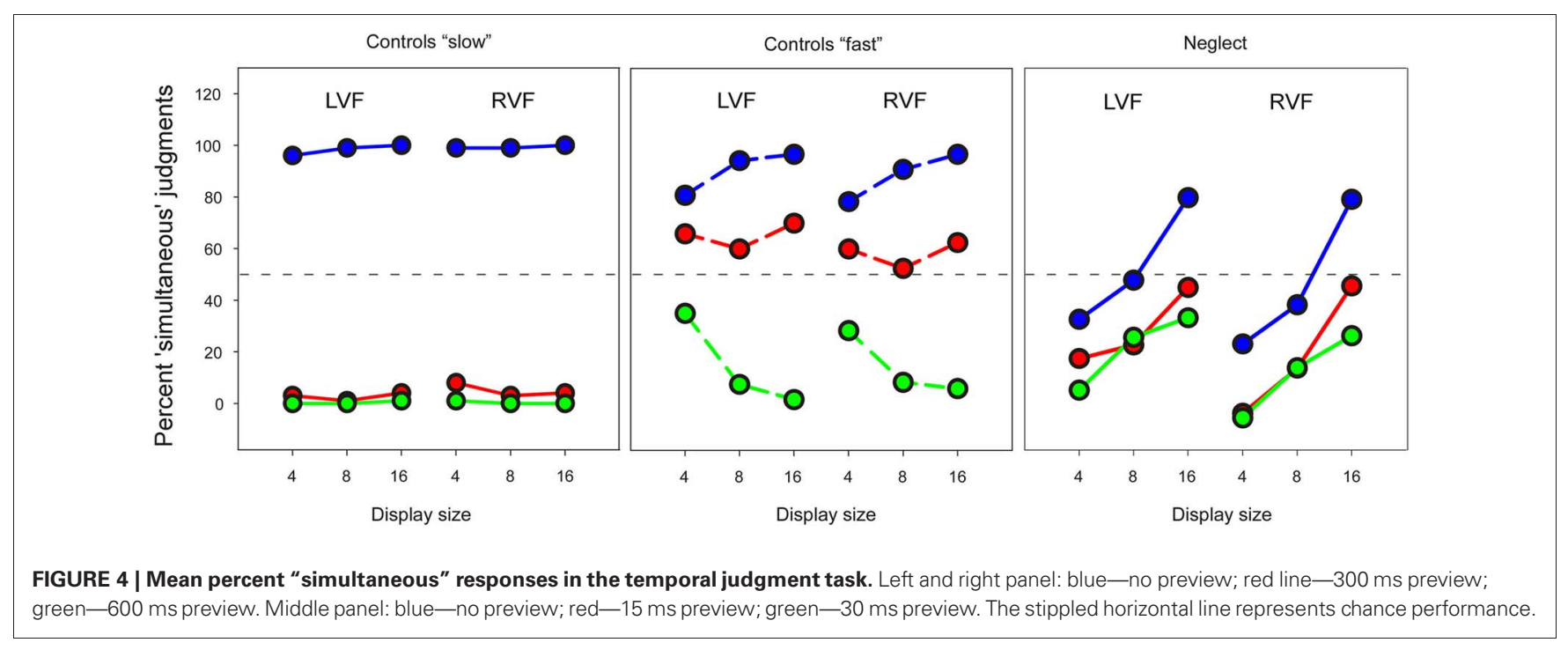


hemifield. In contrast, for left hemifield targets their search data were characterized by two major trends. First, when all search items were presented simultaneously patients showed increasing RTs with increasing numbers of distracters, which is compatible with previous conjunction search studies (Eglin et al., 1989; Esterman et al., 2000; Behrmann et al., 2004). More importantly, though search rates were strongly reduced in the preview conditions neglect patients' visual search remained inefficient at $300 \mathrm{~ms}$ preview, and partly even at $600 \mathrm{~ms}$ preview, when the target appeared in the LVF. Thus, while neglect patients benefited from item preview of LVF items, previewing did not reduce the search task to a pop-out task.

The preview search task used in our study resembles a paradigm used in previous reports to examine a phenomenon known as visual marking, thought to result from top-down inhibition of a subset of distracters presented prior to the rest of the display (preview display; Watson and Humphreys, 1997). However, in a visual marking experiment the preview display only contains distracters and the search target is shown in the final display. By contrast, in our study the preview display contained the target and the final display only distracters. Though the methodological difference appears to be small, the processing requirements of the two paradigms are different: in the visual marking paradigm, search is faster because previewed items are inhibited and search is restricted to items appearing in the final display. In our paradigm, search is restricted to the subset of items presented during preview (provided the deployment of attention during preview is sufficiently fast), resulting in a popout effect. If the target is not found during the preview period attention may drift away due to capture by the upcoming final display.

One might be tempted to explain the failure of neglect patients to show pop-out search in the preview conditions by impaired explicit judgment of temporal simultaneity. Indeed, neglect patients exhibit deficits suggesting an altered perception of time intervals and of the temporal order of events (Becchio and Bertone, 2006). Thus, neglect patients have deficits perceiving the duration of stimuli both for intervals below one second (Basso et al., 1996) and up to 60 s (Danckert et al., 2007). More relevant to the present study is the observation that in situations of asynchronous presentation, a contralesional stimulus must be presented with substantial lead in order to be perceived by neglect patients as simultaneous to an ipsilesional item (Rorden et al., 1997; Robertson et al., 1998). Here, we used a similar paradigm, but varied the display size. The results of the temporal judgment task-though influenced by a response bias (patients made more "simultaneous" judgments with increasing display sizes) revealed two important findings that are helpful in identifying the processes involved in visual search: First, the number of "simultaneous" judgments was not significantly different in the preview compared to the no-preview condition. Second, in contrast to visual search the temporal judgment deficit was independent of target side. These observations are based on a limited number of participants who did not complete the search tasks. Although they suggest that the effect of item preview on visual search is not dependent on explicit knowledge of temporal order, a more confident conclusion to this effect must await a study in which a sufficient number of patients and controls are tested on both search and temporal processing tasks.

Our findings are better explained by impaired deployment of spatial attention in neglect. Cognitive and computational models of visual search distinguish two stages of visual processing (Treisman and Gelade, 1980; Treisman and Gormican, 1988; Wolfe, 1994; Itti et al., 1998): a feature stage, at which individual features are analyzed in separate visual maps, and a conjunction stage, at which features are combined to form spatially coherent objects. According to these models spatially focused attention is necessary for the binding of individual features and the prioritization of important objects. Neurophysiological studies have shown that the activity of neurons in the PPC reflects a combination of bottom-up saliency information and top-down signals carrying information about the behavioral relevance of a stimulus (Gottlieb et al., 1998; Constantinidis and Steinmetz, 2001; Bisley and Goldberg, 2010). Other characteristics of the PPC_-such as feature-independent coding of information and integration of inputs from several modalities-suggest that this region contains a priority map of the environment (Bisley and Goldberg, 2010; Ptak, 2011; Vandenberghe et al., 2012). Recent evidence from lesion studies supports this conclusion. Whereas several reports localized the greatest lesion overlap associated with neglect in the inferior parietal (Vallar and Perani, 1986; Mort et al., 2003; Golay et al., 2008), superior temporal (Karnath et al., 2004) or premotor and ventral frontal cortex (Rengachary et al., 2011), some have found that damage to the PPC is a predictor of specific attention deficits in neglect. Thus, damage affecting the intraparietal sulcus impairs processing of contralesional targets under bilateral stimulation (Vandenberghe et al., 2005), contributes to the appearance of object-based deficits (Ptak et al., 2011), and predicts deficits of attentional shifting and target selection in patients with neglect (Ptak and Schnider, 2011). Moreover, damage to the superior longitudinal fasciculus, which is a major fiber tract connecting the PPC with lateral premotor cortex (Schmahmann and Pandya, 2006), is a predictor of the occurrence of spatial neglect (Thiebaut de Schotten et al., 2005; Bartolomeo et al., 2007), of the preference for ipsilesional locations that characterizes this disorder (Bourgeois et al., 2012), and of the degree to which relevant stimuli capture attention of neglect patients (Ptak and Schnider, 2010). Together, these findings indicate that a frontoparietal network involving the PPC and premotor cortex is crucially involved in the elaboration and representation of attentional priority.

The question arising from these findings is how the failure of neglect patients to fully benefit from item preview can be accommodated with the idea of a parietal priority map. An influential theoretical position holds that neglect results from a spatial selection bias favoring ipsilesional information (Desimone and Duncan, 1995; Duncan, 2004). Applied to the visual search task this hypothesis postulates that due to the right-hemispheric brain damage of patients with neglect ipsilesional items have a higher level of priority than contralesional items. Priority is of importance when search is effortful and requires relatively focused examination of individual items. Therefore, ipsilesional stimuli are found faster when all stimuli are presented simultaneously (conjunction search) because search proceeds from right to left 
according to the degree of priority. In both preview conditions visual search is effortless (feature search) only during the preview period, and attentional priority is less biased in favor of right-sided information. However, in these conditions an additional bias likely affects performance, which is the abrupt onset of additional items at the end of the preview period. Visual onsets capture attention (Yantis and Jonides, 1990) and they do so particularly strongly in patients with lateralized deficits of spatial attention (de Renzi et al., 1989; D'Erme et al., 1992). Right-sided abrupt onsets may therefore delay the activation of search mechanisms or interrupt the on-going search of neglect patients for a left-sided item.

\section{REFERENCES}

Aglioti, S., Smania, N., Barbieri, C., and Corbetta, M. (1997). Influence of stimulus salience and attentional demands on visual search patterns in hemispatial neglect. Brain Cogn. 34, 388-403.

Ashbridge, E., Walsh, V., and Cowey, A. (1997). Temporal aspects of visual search studied by transcranial magnetic stimulation. Neuropsychologia 35, 1121-1131.

Bartolomeo, P., Thiebaut de Schotten, M., and Doricchi, F. (2007). Left unilateral neglect as a disconnection syndrome. Cereb. Cortex 17, 2479-2490.

Basso, G., Nichelli, P., Frassinetti, F., and di Pellegrino, G. (1996). Time perception in a neglected space. Neuroreport 7, 2111-2114.

Becchio, C., and Bertone, C. (2006). Time and neglect: abnormal temporal dynamics in unilateral spatial neglect. Neuropsychologia 44, 2775-2782.

Behrmann, M., Ebert, P., and Black, S. E. (2004). Hemispatial neglect and visual search: a large scale analysis. Cortex 40, 247-263.

Bisley, J. W., and Goldberg, M. E. (2010). Attention, intention, and priority in the parietal lobe. Annu. Rev. Neurosci. 33, 1-21.

Bonato, M., Priftis, K., Marenzi, R., and Zorzi, M. (2009). Normal and impaired reflexive orienting of attention after central nonpredictive cues. J. Cogn. Neurosci. 21, 745-759.

Bourgeois, A., Chica, A. B., Migliaccio, R., de Schotten, M. T., and Bartolomeo, P. (2012). Cortical control of inhibition of return: evidence from patients with inferior parietal damage and visual neglect. Neuropsychologia 50, 800-809.

Bricolo, E., Gianesini, T., Fanini, A., Bundesen, C., and Chelazzi, L. (2002). Serial attention mechanisms in visual search: a direct behavioral demonstration. J. Cogn. Neurosci. 14, 980-993.
Cohen, A., and Rafal, R. D. (1991). Attention and feature integration: illusory conjunctions in a patient with a parietal lobe lesion. Psychol. Sci. 2, 106-110.

Constantinidis, C., and Steinmetz, M. A. (2001). Neuronal responses in area $7 \mathrm{a}$ to multiple-stimulus displays. I. Neurons encode the location of the salient stimulus. Cereb. Cortex 11, 581-591.

Corbetta, M., Shulman, G. L., Miezin, F. M., and Petersen, S. E. (1995). Superior parietal cortex activation during spatial attention shifts and visual feature conjunction. Science 270, 802-805.

D’Erme, P., Robertson, I., Bartolomeo, P., Daniele, A., and Gainotti, G. (1992). Early rightwards orienting of attention on simple reaction time performance in patients with leftsided neglect. Neuropsychologia 30, 989-1000.

Danckert, J., Ferber, S., Pun, C., Broderick, C., Striemer, C., Rock, S., and Stewart, D. (2007). Neglected time: impaired temporal perception of multisecond intervals in unilateral neglect. J. Cogn. Neurosci. 19, 1706-1720.

de Renzi, E., Gentilini, M., Faglioni, P., and Barbieri, C. (1989). Attentional shift towards the rightmost stimuli in patients with left visual neglect. Cortex 25, 231-237.

Desimone, R., and Duncan, J. (1995). Neural mechanisms of selective visual attention. Annu. Rev. Neurosci. 18, 193-222.

Duncan, J. (2004). "Selective attention in distributed brain systems," in Cognitive Neuroscience of Attention, eds M. I. Posner (New York, NY: Guilford Press), 105-113.

Eglin, M., Robertson, L. C., and Knight, R. T. (1989). Visual search performance in the neglect syndrome. J. Cogn. Neurosci. 1, 372-385.

Eramudugolla, R., and Mattingley, J. B. (2009). Spatial gradient for

In conclusion, the present findings suggest two additive processes that contribute to the deficit of neglect patients in conjunction search tasks: a biased representation of attentional priority in favor of ipsilesional information and exaggerated capture of attention by ipsilesional abrupt onsets. The preview paradigm provides a tool for the investigation of distinct attentional processes contributing to impaired search performance in spatial neglect.

\section{ACKNOWLEDGMENTS}

Study supported by the Swiss National Science Foundation (grant 320030-134591).

unique-feature detection in patients with unilateral neglect: evidence from auditory and visual search. Neurocase 15, 24-31.

Esterman, M., McGlinchey-Berroth, R., and Milberg, W. (2000). Preattentive and attentive visual search in individuals with hemispatial neglect. Neuropsychology 14, 599-611.

Friedman-Hill, S. R., Robertson, L. C., and Treisman, A. (1995). Parietal contributions to visual feature binding: evidence from a patient with bilateral lesions. Science 269, 853-855.

Gauthier, L., Dehaut, F., and Joanette, Y. (1989). The Bells Test: A quantative and qualitative test for visual neglect. Int. J. Clin. Neuropsychol. 11, 49-54.

Golay, L., Hauert, C. A., Greber, C., Schnider, A., and Ptak, R. (2005). Dynamic modulation of visual detection by auditory cues in spatial neglect. Neuropsychologia 43 , 1258-1265.

Golay, L., Schnider, A., and Ptak, R. (2008). Cortical and subcortical anatomy of chronic spatial neglect following vascular damage. Behav. Brain Funct. 4, 43.

Gottlieb, J., Kusunoki, M., and Goldberg, M. E. (1998). The representation of visual salience in monkey parietal cortex. Nature 391, 481-484.

Grabowecky, M., Robertson, L. C., and Treisman, A. (1993). Preattentive processes guide visual search: evidence from patients with unilateral visual neglect. J. Cogn. Neurosci. 5, 288-302.

Halligan, P. W. and Marshall, J. C. (1993). "The history and clinical presentation of neglect," in Unilateral Neglect: Clinical and Experimental Studies, eds I. H. Robertson and J. C. Marshall (Hove: Lawrence Erlbaum), 3-25.

Humphreys, G. W. and Riddoch, M. J. (1993). "Interactive attentional systems and unilateral visual neglect," in Unilateral Neglect: Clinical and Experimental Studies, eds I. H. Robertson and J. C. Marshall (Hove: Lawrence Erlbaum), 139-167.

Itti, L., Koch, C., and Niebur, E. (1998). A model of saliency-based visual attention for rapid scene analysis. IEEE Trans. Pattern Anal. Mach. Intell. 20, 1254-1259.

Karnath, H. O., Fruhmann Berger, M., Küker, W., and Rorden, C. (2004). The anatomy of spatial neglect based on voxelwise statistical analysis: a study of 140 patients. Cereb. Cortex 14, 1164-1172.

Milner, A. D., and McIntosh, R. D. (2005). The neurological basis of visual neglect. Curr. Opin. Neurol. 18, 748-753.

Morrow, L. A., and Ratcliff, G. (1988). The disengagement of covert attention and the neglect syndrome. Psychobiology 16, 261-269.

Mort, D. J., Malhotra, P., Mannan, S. K., Rorden, C., Pambakian, A., Kennard, C., and Husain, M. (2003). The anatomy of visual neglect. Brain 126, 1986-1997.

Olivers, C. N., and Humphreys, G. W. (2004). Spatiotemporal segregation in visual search: evidence from parietal lesions. J. Exp. Psychol. Hum. Percept. Perf. 30, 667-688.

Pavlovskaya, M., Ring, H., Groswasser, Z., and Hochstein, S. (2002). Searching with unilateral neglect. J. Cogn. Neurosci. 14, 745-756.

Peru, A., and Chelazzi, L. (2008). Local (focussed) and global (distributed) visual processing in hemispatial neglect. Exp. Brain Res. 187, 447-457.

Posner, M. I., Walker, J. A., Friedrich, F. J., and Rafal, R. D. (1984). Effects of parietal injury on covert orienting of attention. J. Neurosci. 4, 1863-1874.

Ptak, R. (2011). The frontoparietal attention network of the human brain: action, saliency, and a 
priority map of the environment. Neuroscientist doi: 10.1177/ 1073858411409051. [Epub ahead of print].

Ptak, R., Di Pietro, M., and Schnider, A. (2011). The neural correlates of object-centered processing in reading: a lesion study of neglect dyslexia. Neuropsychologia doi: 10.1016/j.neuropsychologia. 2011.09.036. [Epub ahead of print].

Ptak, R., and Schnider, A. (2006). Reflexive orienting in spatial neglect is biased towards behaviorally salient stimuli. Cereb. Cortex 16, 337-345.

Ptak, R., and Schnider, A. (2010). The dorsal attention network mediates orienting toward behaviorally relevant stimuli in spatial neglect. J. Neurosci. 30, 12557-12565.

Ptak, R., and Schnider, A. (2011). The attention network of the human brain: relating structural damage associated with spatial neglect to functional imaging correlates of spatial attention. Neuropsychologia 49, 3063-3070.

Ptak, R., Schnider, A., Golay, L., and Müri, R. (2007). A non-spatial bias favoring fixated stimuli revealed in patients with spatial neglect. Brain 130, 3211-3222.

Ptak, R., and Valenza, N. (2005). The inferior temporal lobe mediates distracter-resistant visual search of patients with spatial neglect. J. Cogn. Neurosci. 17, 788-799.

Rengachary, J., He, B. J., Shulman, G. L., and Corbetta, M. (2011).
A behavioral analysis of spatial neglect and its recovery after stroke. Front. Hum. Neurosci. 5:29. doi: 10.3389/fnhum.2011.00029

Riddoch, M. J., and Humphreys, G. W. (1987). "Perceptual and action systems in unilateral visual neglect." in Neurophysiological and Neuropsychological Aspects of Spatial Neglect, ed M. Jeannerod (NorthHolland: Elsevier), 151-181.

Robertson, I. H., Mattingley, J. B., Rorden, C., and Driver, J. (1998). Phasic alerting of neglect patients overcomes their spatial deficit in visual awareness. Nature 395, 169-172.

Rorden, C., Mattingley, J. B., Karnath, H. -O, and Driver, J. (1997). Visual extinction and prior entry: impaired perception of temporal order with intact motion perception after unilateral parietal damage. Neuropsychologia 35, 421-433.

Schenkenberg, T., Bradford, D. C., and Ajax, E. T. (1980). Line bisection and unilateral visual neglect in patients with neurologic impairment. Neurology 30, 509-517.

Schmahmann, J. D., and Pandya, D. N. (2006). Fiber Pathways of the Brain. Oxford: Oxford University Press.

Schnider, A., Blanche Durbec, V., and Ptak, R. (2011). Absence of visual feedback abolishes expression of hemispatial neglect in self-guided spatial completion. J. Neurol. Neurosurg. Psychiatr. 82, 1279-1282.
Thiebaut de Schotten, M., Urbanski, M., Duffau, H., Volle, E., Lévy, R., Dubois, B., and Bartolomeo, P. (2005). Direct evidence for a parietal-frontal pathway subserving spatial awareness in humans. Science 309, 2226-2228.

Treisman, A., and Gelade, G. (1980). A feature-integration theory of attention. Cogn. Psychol. 12, 97-136.

Treisman, A., and Gormican, S. (1988). Feature analysis in early vision: evidence from search asymmetries. Psychol. Rev. 95, 15-48.

Vallar, G., and Perani, D. (1986). The anatomy of unilateral neglect after right-hemisphere stroke lesions. A clinical/CT-scan correlation study in man. Neuropsychologia 24, 609-622.

Vandenberghe, R., Geeraerts, S., Molenberghs, P., Lafosse, C., Vandenbulcke, M., Peeters, K., Peeters, R., van Hecke, P., and Orban, G. A. (2005). Attentional responses to unattended stimuli in human parietal cortex. Brain 128, 2843-2857.

Vandenberghe, R., Molenbergs, P., and Gillebert, C. R. (2012). Spatial attention deficits in humans: the critical role of superior compared to inferior parietal lesions. Neuropsychologia doi: $\quad 10.1016 /$ j.neuropsychologia. 2011.12.016. [Epub ahead of print].

Watson, D. G., and Humphreys, G. W. (1997). Visual marking: prioritizing selection for new objects by top-down attentional inhibition of old objects. Psychol. Rev. 104, 90-122.

Wilson, B., Cockburn, J., and Halligan, P. (1987). Behavioral Inattention Test. Bury St Edmunds: Thames Valley Test Company.

Wolfe, J. M. (1994). Guided Search 2.0. A revised model of visual search. Psychonom. Bull. Rev. 1, 202-238.

Yantis, S., and Jonides, J. (1990). Abrupt visual onsets and selective attention: voluntary versus automatic allocation. J. Exp. Psychol.Hum. Percept. Perf. 16, 121-134.

Conflict of Interest Statement: The authors declare that the research was conducted in the absence of any commercial or financial relationships that could be construed as a potential conflict of interest.

Received: 30 January 2012; accepted: 31 March 2012; published online: 18 April 2012.

Citation: Fellrath J, Blanche-Durbec V, Schnider A, Jacquemoud A-S and Ptak $R$ (2012) Visual search in spatial neglect studied with a preview paradigm. Front. Hum. Neurosci. 6:93. doi: 10.3389/ fnhum.2012.00093

Copyright (c) 2012 Fellrath, BlancheDurbec, Schnider, Jacquemoud and Ptak. This is an open-access article distributed under the terms of the Creative Commons Attribution Non Commercial License, which permits non-commercial use, distribution, and reproduction in other forums, provided the original authors and source are credited. 